\title{
Der Einfluß der Austauschenergie auf die spezifische Wärme des Elektronengases
}

\author{
Von Heinz Koppe \\ Aus dem Kaiser-Wilhelm-Institut für Physik in Göttingen \\ (Z. Naturforschg. 2a, 429-432 [1947]; eingegangen am 5. März 1947)
}

\begin{abstract}
Es wird der Einfluß der durch die Antisymmetrisierung der Eigenfunktionen bedingten Austauschenergie auf die spezifische Wärme des Elektronengases untersucht. Es ergibt sich, daß, abgesehen von extrem kleinen Temperaturen, die Proportionalität von $C_{v}$ zu $T$ erhalten bleibt, nur ist der Proportionalitätsfaktor bis zu $50 \%$ kleiner als in der Sommerfeldschen Theorie.
\end{abstract}

$\mathrm{D}$ ie bekannte Formel für die spezifische Wärme des Elektronengases ist unter der Voraussetzung abgeleitet, daß die Elektronen dem PauliPrinzip genügen und man es demzufolge mit einem entarteten Fermi-Dirac-Gas zu tun hat. Von der Ladung der Elektronen wird dabei völlig abgesehen. Solange man die Eigenfunktionen als ebene Wellen betrachtet, bekommt man infolge der wechselseitigen Abstoßung eine positive potentielle Energie, die durch die Anziehung zwischen den Elektronen und den Kernen zum größten Teil kompensiert wird, und die im übrigen von der Geschwindigkeitsverteilung unabhängig ist, also auf die thermodynamischen Eigenschaften des Elektronengases keinen Einfluß hat. Dabei ist aber die notwendige Antisymmetrisierung der Eigenfunktionen außer acht gelassen worden, die bewirkt, daß sich Elektronen vom gleichen Spin gegenseitig etwas ausweichen. Auf diese Weise entsteht um jedes Elektron ein „Austauschloch“, und der Beitrag dieses Loches zur potentiellen Energie ist von der Gesamtenergie wieder abzuziehen. Die Rechnung, die von $\mathrm{Bloch}{ }^{1}$ durchgeführt wurde, führt zu dem bekannten Ausdruck für die Austauschenergie.

Wie weit sich zwei Elektronen infolge des Pauli-Prinzips gegenseitig ausweichen, hängt aber wesentlich von ihrer Relativgeschwindigkeit ab. Die Austauschenergie $B$ ist also gegenüber der Geschwindigkeitsverteilung durchaus nicht unempfindlich. Man erkennt das am einfachsten an

\footnotetext{
1 F. B l o c h, Z. Physik 57, 545 [1929].
}

dem Ausdruck²

$$
B=\frac{1}{2} \sum_{i=1=k} \frac{h^{2} e^{2}}{\pi V} \frac{1}{\left|p_{i}-p_{k}\right|^{2}} .
$$

Dabei ist ${ }^{*} V$ das Normierungsvolumen, $p_{i}$ der Impuls des $i$-ten Elektrons und die Summation ist über alle Elektronenpaare zu erstrecken. Man kann aus (1) ohne weiteres ablesen, daß die Austauschenergie zu einer Art „Anziehung im Impulsraum" führt, die die Tendenz hat, alle Phasenpunkte auf einem Haufen zu versammeln. Sie wirkt der ausbreitenden Tendenz der Wärmebewegung entgegen und muß deshalb zu einer Verkleinerung der spezifischen Wärme führen. Gerade bei tiefen Temperaturen wird dieser Einfluß besonders stark sein. Denn hier kommt ein angeregtes Elektron aus der dichtgepackten Oberfläche der FermiKugel, wo es also eine große Austauschenergie hat, in die wesentlich dünner besiedelten angeregten Zustände. Dazu muß es dann nicht nur den entsprechenden Betrag an kinetischer Energie aufbringen, sondern auch noch gegen die Austauschenergie Arbeit leisten.

Im folgenden wird die Rechnung zunächst für freie Elektronen gleichen Spins durchgeführt. Um zur Verteilungsfunktion zu gelangen, hat man den Ausdruck für freie Energie aufzustellen und bei der Nebenbedingung konstanter Teilchenzahl zu einem Minimum zu machen. Wenn $n(p)$ die (nur vom Betrage $p$ abhängige) Besetzungșwahrschein-

2 Vergl. z. B. H. Hellm an n, Quantenchemie, Leipzig 1937, S. $101 \mathrm{ff}$. 
lichkeit einer Quantenzelle im Impulsraum ist, dann ergibt sich

$$
\begin{gathered}
F=E-B-k T \cdot S=\frac{4 \pi V}{h^{3}} \frac{1}{2 m} \int_{0}^{\infty} n(p) p^{4} d p \\
-\frac{4 \pi V}{h^{3}} \cdot \frac{e^{2}}{h} \int d p_{1} \int d p_{2} p_{1} n\left(p_{1}\right) p_{2} n\left(p_{2}\right) \log \frac{p_{1}+p_{2}}{\left|p_{1}-p_{2}\right|} \\
-k T \frac{4 \pi V}{h^{3}} \int p^{2} d p\{n \log n-(1-n) \log (1-n)\}, \\
N=\frac{4 \pi V}{h^{3}} \int_{0}^{\infty} n(p) \cdot p^{2} d p .
\end{gathered}
$$

Das Doppelintegral stellt dabei die Austauschenergie dar und ergibt sich aus (1), wenn man die Summation durch eine Integration ersetzt und über die Winkel integriert. Führt man die Variation unter Einführung des Multiplikators $\zeta$ für die Nebenbedingung durch, dann ergibt sich für $n(p)$ die Funktionalgleichung

$$
\begin{aligned}
\frac{1}{2 m} p^{2}-\frac{2 e^{2}}{h} & \cdot \frac{1}{p} \int_{0}^{\infty} p^{\prime} n\left(p^{\prime}\right) \log \frac{p+p^{\prime}}{\left|p-p^{\prime}\right|} d p^{\prime}-\zeta \\
& =k T \ln \frac{1-n(p)}{n(p)} .
\end{aligned}
$$

Leider ist sie so kompliziert, daß mit ihr nichts anzufangen ist. Wir wollen des Problem deshalb annähernd nach der direkten Methode der Variationsrechnung behandeln. Die zu diesem $Z_{w e c k}$ benutzte Näherungsfunktion für $n(p)$ ist in Abb.1 dargestellt. Analytisch ist sie gegeben durch:

$$
\begin{array}{lrl}
n(p)=1 & \text { für } 0 \leq p \leq a \\
n(p)=a-\beta p & a \leq p \leq b \\
n(p)=0 & b \leq p .
\end{array}
$$

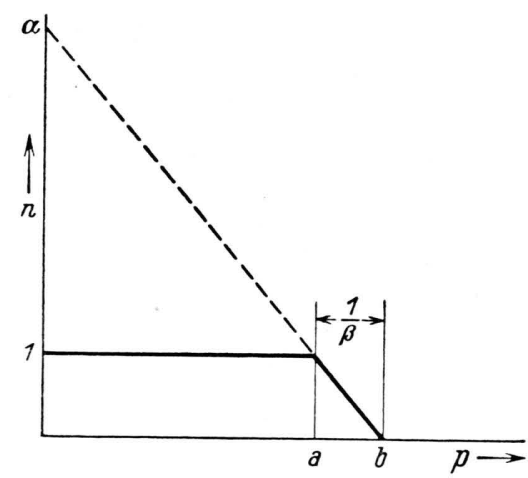

Abb. 1. Näherungsfunktion fuir $n(p)$. $a$ und $b$ oder $\alpha$ und $\beta$ sind Variationsparameter. Die beiden letzteren erweisen sich als die zweckmäßigeren, und es gilt:

$$
a=\frac{a-1}{\beta} ; \quad b=\frac{a}{\beta} .
$$

Aus der Abb. 1 erkennt man sofort, daß $\propto$ sehr groß und $1 / \alpha$ proportional zur Temperatur sein muß. Da es bei der spezifischen Wärme auf Glieder $o\left(T^{2}\right)$ ankommt, braucht man also in der Folge nur bis zu Gliedern $o\left(\alpha^{-2}\right)$ zu rechnen. Es ergibt sich auf diese Weise:

$$
\begin{gathered}
E=\frac{4 \pi V}{3 h^{3}} \frac{3}{10 m}\left(\frac{a}{\beta}\right)^{5}\left(1-\frac{5}{2} a^{-1}\right. \\
\left.\quad+\frac{10}{3} a^{-2}+\ldots\right), \\
B=\frac{4 \pi V}{3 h^{3}} \cdot \frac{3}{2} \frac{e^{2}}{h}\left(\frac{a}{\beta}\right)^{4}\left(1-2 \alpha^{-1}\right. \\
\left.\quad-\frac{59}{4} a^{-2}-\frac{5}{6} \alpha^{-2} \log 2 a\right), \\
\log W=\frac{4 \pi V}{3 h^{3}} \cdot \frac{3}{2}\left(\frac{\alpha}{\beta}\right)^{3} \cdot \alpha^{-1}+\ldots, \\
N=\frac{4 \pi V}{3 h^{3}}\left(\frac{\alpha}{\beta}\right)^{3}\left(1-\frac{3}{2} \alpha^{-1}+a^{-2}+\ldots\right) .
\end{gathered}
$$

Indem man die ersten drei Ausdrücke je durch eine passende Potenz von $N$ dividiert, kann man $\beta$ und damit die Nebenbedingung (3) eliminieren. Man findet dann für die freie Energie pro Teilchen

$$
\begin{gathered}
\frac{F}{N}=\left(\frac{3}{4 \pi}\right)^{2 / 3} \frac{3 h^{2}}{10 m} n^{2 / 3}\left(1+\frac{5}{12} x^{2}\right) \\
-\left(\frac{3}{4 \pi}\right)^{1 / 3} \frac{3 e^{2}}{2} n^{1 / 3}\left(1-\frac{199}{12} x^{2}\right. \\
\left.+\frac{5}{6} x^{2} \log \frac{x}{2}\right)-\frac{3}{2} k T x .
\end{gathered}
$$

Dabei ist $\alpha^{-1}=x$ gesetzt, und $n$ ist die Anzahl der Elektronen pro Volumeneinheit. Man drückt sie zweckmäßig durch das „Elektronenvolumen“ $V_{e}$ aus, welches gegeben ist durch

$$
n=\frac{N_{L}}{V_{e}} .
$$

$V_{e}$ ist dann ein Volumen von einigen $\mathrm{cm}^{3}$. Jetzt ist $F$ als Funktion von $x$ zum Minimum zu machen. 
Das liefert mit den Abkürzungen

$$
\begin{gathered}
\vartheta=6\left(\frac{4 \pi}{3}\right)^{2 / 3} \frac{k \cdot m}{h^{2} N_{L}^{2 / 3}} V_{e}^{2 / 3} T \\
=3,145 \cdot 10^{-5} V_{e}^{2 / 3} T, \\
q=6\left(\frac{4 \pi}{3}\right)^{1 / 3} \frac{e^{2} m}{h^{2} N_{L}^{1 / 3}} V_{e}^{1 / 3}=0,1167 V_{e}^{1 / 3}
\end{gathered}
$$

für $x$ die Bestimmungsgleichung

$$
\vartheta=(1+q) x-\frac{5}{97} q x \log \frac{x}{2} .
$$

Daraus kann man $x$ als Funktion von $\vartheta$ bzw. $T$ berechnen und den gefundenen Wert in (7) einsetzen, womit die freie Energie und damit alle thermodynamischen Größen bekannt sind.

Die spezifische Wärme bekommt man aber einfacher so:

$$
C_{v}=N_{L} \frac{\partial}{\partial T} \frac{U}{N}=N_{L} \frac{\partial}{\partial x} \frac{U}{N} \cdot \frac{d x}{d \vartheta} \cdot \frac{d \vartheta}{d T} ;
$$

anderseits ist

$$
\frac{\partial}{\partial x} \frac{F}{N}=\frac{\partial}{\partial x}\left(\frac{U}{N}-T \frac{S}{N}\right)=0,
$$

also wegen $(6,3)$

$$
\frac{\partial}{\partial x} \frac{U}{N}=\frac{3}{2} k^{\prime} T^{\prime} .
$$

Die beiden anderen Differentialquotienten bekommt man aus $(9,1)$ bzw. (10), und es ergibt sich

$$
C_{v}=\frac{9\left(\frac{4 \pi}{3}\right)^{2 / 3} k^{2} h^{-2} m N_{L}^{1 / 3} V_{e}^{2 / 3}}{1+q\left(\frac{92}{97}-\frac{5}{97} \ln \frac{x}{2}\right)} T .
$$

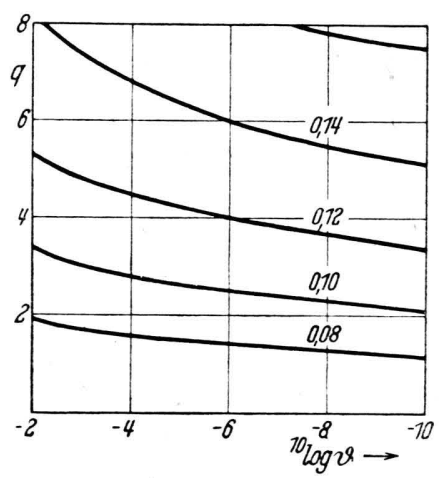

Abb. 2. Diagramm zur Ermittlung von $\psi$ mit $\psi=$ const. als Parameter.
Hier ist noch $x$ mittels Gl. (10) zu eliminieren, man kann dann schreiben:

$$
C_{v}=\frac{9\left(\frac{4 \pi}{3}\right)^{2 / 3} h^{-2} k^{2} m N_{L}^{1 / 3} V_{e}^{2 / 3}}{1+q\left(0,9905-0,120^{10} \log \vartheta+\psi\right)} \cdot T^{\prime} .
$$

Dabei ist $\psi$ eine langsam veränderliche Funktion von $\vartheta$ und $q$, die aus dem Diagramm Abb. $2 \mathrm{zu}$ entnehmen ist.

Setzt man in (11) $q=0$, so bekommt man die spezifische Wärme $C_{v}^{0}$ ohne Berücksichtigung des Austausches. Natürlich wird sich nicht der genaue Wert ergeben, da ja die ganze hier gegebene Ableitung auf einem Näherungsverfahren beruht. Ein Vergleich mit dem entsprechenden Wert der Sommerfeldschen Theorie ${ }^{3}$ zeigt, daß an Stelle des Faktors 9 ,im Zähler $\pi^{2}=9,87$ treten muß. Durch das Näherungsverfahren ist also ein Fehler von etwa $10 \%$ entstanden. Wir können ihn weitgehend ausschalten, wenn wir für die spezifische Wärme des Elektronengases ohne Austausch wie oben $C_{v}^{\prime \prime}$ schreiben, und $(11,2)$ in die Form

$\frac{C_{v}^{0}}{C_{v}}=1+q\left(0,9905-0,120{ }^{10} \log \vartheta+\psi\right)$

bringen.

Bevor wir an die Diskussion dieses Ergebnisses gehen, ist noch eine zweite Korrektur zu erörtern. Die bisherigen Rechnungen galten nur für Elektronen mit gleichem Spin. Der Übergang zu einem Elektronengas mit paarweise abgesättigten Spins kann in der bekannten Weise gemacht werden, indem man einfach für $V_{e}$ den doppelten Wert einsetzt. Allerdings ist dieses Verfahren nur dann gerechtfertigt, wenn sich die Elektronen mit entgegengesetztem Spin nicht umeinander kümmern. In Wirklichkeit besteht nun auch zwischen diesen eine Wechselwirkung, die natürlich nicht vom $\mathrm{Pa}$ a li-Prinzip, sondern von der gegenseitigen Abstoßung herrührt. Sie ist von Wigner ${ }^{4}$ untersucht worden und statistisch schwer zu berücksichtigen, da sie sich nicht in Analogie zu (1) durch "Zentralkräfte“ darstellen läßt.

3 Vergl. A. Sommerfeld und H. Bethe, Handbuch der Physik, XXIV, 2, S. 347.

${ }^{4}$ E. Wigner, Physic. Rev. 46, 1002 [1934]. 
Betrachtet man nun die rechte Seite von $(11,3)$, so sieht man, daß man den Einfluß der Austauschenergie beliebig groß machen kann, wenn man nur $T$ so klein wählt, daß das logarithmische Glied vorherrscht. Bei extrem tiefen Temperaturen folgt dann aus $(11,3)$, daß

$$
C_{v} \sim V_{e}^{1 / 3} \frac{T}{|\ln T|},
$$

und das unterscheidet sich in seiner Abhängigkeit von $T$ und $V$ schon qualitativ von der Sommerfeldschen Theorie. Jedoch gilt diese Beziehung erst unterhalb von $T \sim 10^{-4}{ }^{\circ} \mathrm{K}$, so daß sie praktisch völlig bedeutungslos ist. Normalerweise stellt der Logarithmus nur eine kleine Korrektur dar, so daß die rechte Seite von $(11,3)$ annähernd temperaturunabhängig ist. $C_{v}$ ist also wie in der Sommerfeldschen Theorie zu $T$ proportional, nur ist der Proportionalitätsfaktor etwas anders, und zwar kleiner.
Rein numerisch fällt dieser Unterschied vor allem bei Metallen mit großem $V_{e}$, also den Alkalien, ins Gewicht. Um einen UUberblick zu bekommen, werten wir $(11,3)$ für $\mathrm{Na}$ und $\mathrm{Cs}$ bei $T=1{ }^{\circ} \mathrm{K}$ aus. Es ergibt sich:

$$
\begin{aligned}
\mathrm{Na}: \quad V_{e} & =20 \mathrm{~cm}^{3} ; \\
q & =0,403 ; \quad \vartheta=3,68 \cdot 10^{-4} \\
C_{v} & =0,633 C_{v}^{0} \\
\mathrm{Cs}: \quad V_{e} & =70 \mathrm{~cm}^{3} \\
q & =0,606 \quad \vartheta=8,5 \cdot 10^{-4} \\
C_{v} & =0,541 C_{v}^{0} .
\end{aligned}
$$

Man erkennt aus diesen Zahlen, daß der Einfluß der Austauschenergie auf die spezifische Wärme der Elektronen, zum mindesten bei den Alkalimetallen, nicht zu vernachlässigen ist.

\title{
Über die nach der Umwandlung von Tantal durch Neutronen ausgesandten Strahlungen mit langer Halbwertszeit
}

\author{
Von Hugo Neuert: \\ (Z. Naturforschg. 2a, 432—435 [1947]; eingegangen am 6. Mai 1947)
}

\begin{abstract}
Die mit einem $\beta$-Zählrohr mit $20 \mu$-Al-Fenster gemessene $\beta$-Strahlung setzt sich aus 2 Komponenten zusammen, einer energieärmeren $\beta$-Strahlung von $0,28 \mathrm{MeV}$ und einer energiereicheren von $1,1 \mathrm{MeV}$. Außerdem treten 2 Komponenten von $\boldsymbol{\gamma}$-Strahlung auf, eine härtere von $1,42 \mathrm{MeV}$ und eine weiche von $0,23 \mathrm{MeV}$. Aus $\beta$ - $\gamma$-Koinzidenzmessungen geht hervor, daß die harte $\beta$-Strahlung gleichzeitig mit der weichen $\gamma$-Strahlung emittiert wird. Sie entstammt dem Prozeß ${ }^{181} \mathrm{Ta}(n, \gamma){ }^{182} \mathrm{Ta}^{*}$. Die weiche $\beta$-Strahlung koinzidiert mit der harten $\gamma$-Strahlung. Sie rührt rom Prozeß ${ }^{181} \mathrm{Ta}(n, p){ }^{181} \mathrm{Hf}^{*}$ her.
\end{abstract}

Z um Studium der langlebigen Strahlung von Tantal wurde ein Tantalblech („rein“, Herstellungsverfahren Osram) während mehrerer Tage mit langsamen und schnellen Neutronen eines Neutronengenerators von maximal etwa $8 \mathrm{~kg} \mathrm{Ra}+\mathrm{Be}-$ Aqquivalent bestrahlt. Nach dem Abklingen der kurzlebigen Aktivität von Tantal (8,2 Stdn.) trat neben der erwarteten intensiven $\beta$-Strahlung von etwa $1,0 \mathrm{MeV}^{1}$ noch eine weitere $\beta$-Strahlung sowie eine intensive $\gamma$-Strahlung auf. Messungen der Halbwertszeiten konnten infolge der zur Verfügung stehenden kurzen Zeit nicht durchgeführt werden. Die Halbwertszeit aller

* (17) Weil (Rh.), Marktplatz 5.
Strahlen war aber mit Sicherheit größer als 50 Tage.

Bestimmung der $\beta$-Strahlenenergie aus Absorptionsmessungen

Es wurde die Absorption der $\beta$-Strahlung in Aluminium mit einem $20 \mu$-Al-Zähler von $10 \mathrm{~cm}$ Länge, $3 \mathrm{~cm}$ Durchmesser und $6 \times 1,5 \mathrm{~cm}^{2}$ Fenstergröße ausgemessen. Nach Abzug des $\gamma$-Anteils blieb eine $\beta$-Strahlung übrig, die sich offensichtlich aus 2 Anteilen zusammensetzte (Abb. 1), nämlich aus der schon bekannten $\beta$-Strahlung,

1 O. Oldenberg, Physic. Rev. 53, 35 [1938]; F. G. Ho utermans, Naturwiss. 28, 578 [1940]. 